\section{A New Interpretation and improvement of the Nonlinear Anisotropic Diffusion for Image Enhancement}

\author{
Jérôme Monteil and Azeddine Beghdadi
}

\begin{abstract}
The purpose of this article is to give a new analysis of the Anisotropic Diffusion $(A D)$ and propose an adaptive nonlinear filtering based on a judicious choice of the Conductance Function (CF) and the edgeness threshold. A new undesirable effect, which we call the "pinhole effect," may result when $A D$ is introduced for the first time. A robust solution to this effect is proposed and evaluated through experimental data. The evolution of the diffused signal is analyzed through a physical model using the Optical Flow Technique (OFT). The overall strategy is evaluated through experimental results obtained on synthetic and actual images.
\end{abstract}

Index Terms-Nonlinear anisotropic diffusion, fluid mechanics, homogeneity, image enhancement, noise smoothing, optical flow.

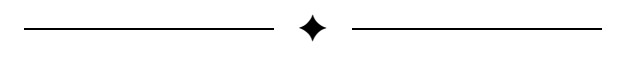

\section{INTRODUCTION}

ONE way to facilitate contour extraction is to design a filter which combines a low pass filtering in homogeneous regions and a sharpening effect in transition regions. However, it is not easy to design a linear filter which achieves these two conflicting goals. This motivates the development of a multitude of nonlinear filters based on order statistics [1], [2], mathematical morphology [3], [4], or Partial Differential Equations (PDEs) related to diffusion process [5], [6], [7]. A nonlinear filter class that has been proven very efficient is the one based on nonlinear anisotropic diffusion. The basic idea is to consider the image signal as a medium where diffusion can take place with a variable conductance [5], [8]. However, some drawbacks and limitations in this model have been mentioned in the literature [9], [10], [11]. Indeed, Catte et al. [9] have proven the ill-posedness, in some cases, of the diffusion equation when using the CF proposed by Perona and Malik. Whitaker and Pitzer have pointed out the staircasing effect that may occur on wide smooth edges when the conductance parameter is not carefully adapted to the range of gradient values [10]. Besides these works, an interesting strategy has been proposed by Li and Chen [11]. The basic idea is to use a decreasing function of the gradient and to adapt the conductance parameter $\kappa$ during the diffusion process evolution. They have also noticed, as did Whitaker and Pizer, that choosing an appropriate single value for $\kappa$ so as to both sharpen the edge and remove the noise is rather a difficult task. The aim of this work is twofold : to give a new look at the AD in the framework of physics and to propose an Adaptive Nonlinear Anisotropic Diffusion (ANAD) for image enhancement. The paper is organized into six sections. After a brief introduction, the anisotropic diffusion model of Perona and Malik is recalled, followed by the improvements suggested by Li and Chen. Section 3 presents a physical interpretation of the AD with the help of OFT and a new image model we introduce, "the corpuscular model." The next section is devoted to the improvements we propose. Section 5 presents the experimental results and the evaluation of

- The authors are with L2Ti-Institut Galilée, Université Paris Nord, Avanue J.B. Clément, 93400 Villetaneuse, France.

E-mail: \{Jerome.Monteil, Azeddine.Beghdadi\}@12ti.univ-paris13.fr.

Manuscript received 23 Oct. 1997; revised 8 June 1999.

Recommended for acceptance by V.S. Nalwa.

For information on obtaining reprints of this article, please send e-mail to: tpami@computer.org, and reference IEEECS Log Number 107748. the proposed method. Concluding remarks and open questions are given in the last section.

\section{Standard Nonlinear Anisotropic Diffusion (SNAD) AND SOME IMPROVEMENTS}

Let us recall Perona and Malik's model, which we call SNAD. The image is considered as a medium where a fluid can diffuse in an anisotropic manner. In this case, the heat equation which governs the temporal evolution of the intensity is given by:

$$
\frac{\partial}{\partial t} I(\mathbf{x}, t)=\nabla^{T}(c(\mathbf{x}, t) \nabla I(\mathbf{x}, t))
$$

where $c(\mathbf{x}, t)$ is the conductance function. The idea of Perona and Malik is to chose a decreasing CF of the gradient in order to insure high diffusion in homogeneous regions and weak diffusion near edges. They suggested two heuristic functions satisfying these requirements:

$$
c_{1}(\mathbf{x}, t)=\exp \left(-\frac{\|\nabla I(\mathbf{x}, t)\|}{\kappa}\right)^{2}
$$

or

$$
c_{2}(\mathbf{x}, t)=\frac{1}{1+(\|\nabla I(\mathbf{x}, t)\| / \kappa)^{1+\alpha}} \quad(\text { where } \alpha>0) .
$$

However, Catté et al. [9] have shown that, for some gradient values, the problem turns to an inverse heat equation which is known to be an ill-posed problem. To overcome this difficulty, another scaling parameter $\sigma$ intervening in an implicit Gaussian filtering is introduced [10], [11]. In the method of Whitaker and Pizer (WP method), a decreasing function of time $\sigma(t)$ is suggested. But, these authors do not give a strategy allowing us to automatically adapt this parameter to the local properties of the image signal. $\mathrm{Li}$ and Chen [11] used the same idea but adopted another strategy, where the conductance parameter $\kappa$ is empirically adapted to the signal activity. In the following sections, we give a strategy which automatically computes the adequate value of $\kappa$. We also experimentally show why the CFs $c_{1}$ and $c_{2}$ are not well adapted in some cases for filtering purpose. To track the diffusion process, we make use of the OFT in the framework of a physical model where the image is considered as a set of particles.

\section{Optical Flow Technique (OFT) as an Aid for DifFUSION TRACKING}

First, in order to make the article self-sufficient, the Standard Optical Flow (SOF) of Horn and Schunck [12] is recalled. We do not use more elaborated OFT [13], which have been proven more efficient than SOF, since the aim of this work is not to evaluate the OFT.

\subsection{Optical Flow and Fluid Dynamics}

The strong hypothesis of SOF is the intensity constraint (IC) which states the intensity conservation during the movement:

$$
I_{t}+u I_{x}+v I_{y}=0,
$$

where $(u, v)$ are the components of the velocity vector $\mathbf{V}$ and the subscribes indicate the derivations with respect to the temporal and spatial variables. Further constraints are necessary to solve for both components of $\mathbf{V}$. Horn and Schunck introduced an additional constraint, called the smoothness constraint (SC), which states that the displacement field is supposed to be smooth. The (IC) and (SC) could be put together and expressed as the following optimization problem: 


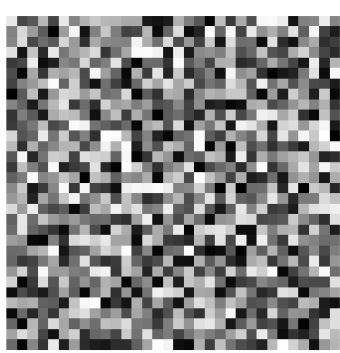

(a)

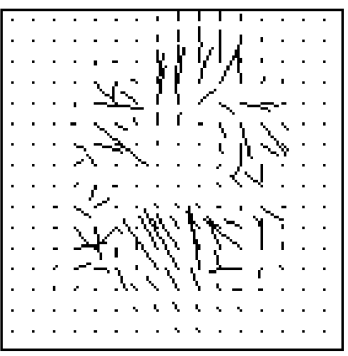

(c)

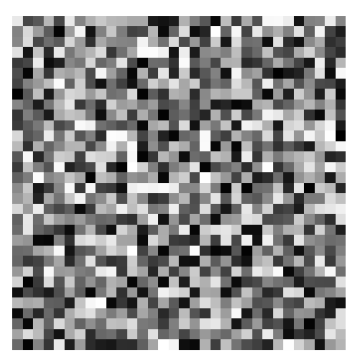

(b)

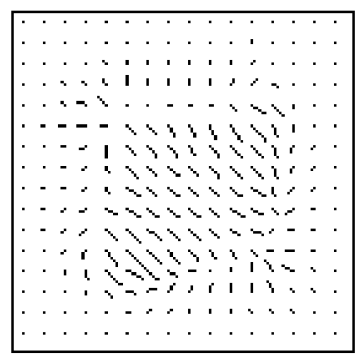

(d)
Fig. 1. Comparison of SOF with OF-GIC. (a) Original image. (b) Image (3a) after diffusion. (c) Flow field obtained with SOF. (d) Flow field obtained with OF-GIC.

$$
\iint_{\Omega}\left\{\left[I_{t}+u I_{x}+v I_{y}\right]^{2}+\alpha^{2}\left[\|\nabla u\|^{2}+\|\nabla v\|^{2}\right]\right\} d \Omega
$$

where $\Omega$ is the local neighborhood where the vector field $\mathbf{V}$ is supposed to be smooth and where $\alpha$ is a parameter allowing to control the weight accorded to the (SC). Using the well-known Euler-Lagrange formalism, an iterative solution is obtained. Schunck has noticed that the IC is equivalent to the equation of continuity derived from the fluid mechanics in the case of a noncompressible fluid [14]. Otherwise, the introduction of the divergence term of $\mathbf{V}^{1}$ yields:

$$
I_{t}+u I_{x}+v I_{y}+\left(u_{x}+v_{y}\right) I=0 .
$$

If we again make the analogy with fluid mechanics, the heat equation (1) could be interpreted as the second law of Fick [15] which governs the diffusion of particles in a fluid. Indeed, if $n(\mathbf{x}, t)$ is the mean density of particles, this law is expressed in the following equation.

$$
\frac{\partial}{\partial t} n(\mathbf{x}, t)=\nabla^{T}[c(\mathbf{x}, t) \nabla n(\mathbf{x}, t)]
$$

where $c$ is the self-diffusion coefficient. Thus, we can see that (6) is nothing but (1) where $I$ is replaced by $n$. Notice that this law follows from the two following relations:

$$
\begin{gathered}
\mathbf{j}=-c(\mathbf{x}, t) \nabla n \\
\frac{\partial}{\partial t} n(\mathbf{x}, t)+\nabla^{T} \mathbf{j}=0,
\end{gathered}
$$

where $\mathbf{j}$ is the current density vector given by:

$$
\mathbf{j}=n(\mathbf{x}, t) \mathbf{V}(\mathbf{x}, t) \text {. }
$$

Replacing $\mathbf{j}$ in (8) by its expression (9), we can see that (8) is equivalent to (5), where $I$ plays the role of $n$. This analogy shows that the diffusion equation (1) of Perona and Malik implicitly contains the IC (5). Therefore, this observation fully justifies the use

1. When we deal with this equation, we refer to Optical Flow with Generalized Intensity Constraint (OF-GIC), while we refer to Standard Optical Flow (SOF) when (3) is used. of the OF-GIC to analyze the diffusion process in image signal.

\subsection{The Corpuscular Image Model (CIM)}

Now, we consider the image as a collection of fictive identical particles embedded in a $2 \mathrm{~d}$ space $\Omega$ of finite size. This set could be discretized into a finite number of nonoverlapping subsets $\omega_{j}$ representing a pixel or a group of adjacent pixels. Let $n(\mathbf{x}, t)$ be the spatio-temporal distribution of the particle density. It is assumed that, in this fictive gas, the particles have the same elementary charge, say g, for example, which could represent a quantum of gray level in the digital image. Then, the intensity measured in the subset $\omega_{j}$ at the instant $t$ is given by:

$$
I_{j}(t)=\int_{\omega_{j}} g n(\mathbf{x}, t) d \omega=g N_{j}(t),
$$

where $N_{j}(t)$ is the number of particles contained in an elementary set $\omega_{j}$ at the instant $t$. Thus, in our physical representation, a "black pixel" is assimilated to a vacuum domain (no particle), whereas a "white pixel" corresponds to a domain with the maximum allowed number of particles. With this formalism, one can define the average instantaneous velocity of the particles contained in the subset $\omega_{j}$, at the location $\mathbf{x}$ by:

$$
V_{j}(\mathbf{x}, t)=\frac{1}{N_{j}(t)} \sum_{k=1}^{N_{j}(t)} V_{k}\left(\omega_{j}, t\right),
$$

where $\mathbf{V}_{k}\left(\omega_{j}, t\right)$ is the individual velocity of the $k$ th fictive particle belonging to $\omega_{j}$ at instant $t$. The use of OF-GIC as a tool for tracking these particles is then plausible. To assert this statement, let us examine the synthetic image of Fig. 1, where a central block of size $16 \times 16$ is submitted to a directional diffusion in a $32 \times 32$ matrix. In this experiment, the diffusion could be interpreted, in the framework of CIM, as a displacement, in the diagonal direction, of fictive particles contained in the central block. In terms of image signal, this diffusion corresponds to an intensity change of +2 . Figs. $1 \mathrm{a}$ and $1 \mathrm{~b}$ show the image before and after diffusion, respectively. Fig. 1c displays the flow field obtained using SOF. The use of OF-GIC leads to the more realistic result shown in Fig. 1d. It could be noticed that, even with the OF-GIC, the flow field is not correctly estimated near the block border. This is due to the regularization constraint, which tends to propagate the smoothness of the flow field. This result confirms the inadequacy of using SOF for computing the flow field and the usefulness of OF-GIC in tracking and visualizing gray level changes as flow field in the framework of the CIM.

\section{Adaptive Nonlinear ANISOtropic Diffusion (ANAD)}

For the sake of simplicity and clarity of the analysis, the main ideas of our filtering method are progressively presented.

\subsection{Analysis of the Conductance Function Behavior}

It could be noticed from Figs. $2 a$ and $2 b$ that the transition length between the maximum and minimum conductance values varies with the parameter $\kappa$. This transition length, or gradient gray-level range, increases with $\kappa$. This makes the control of the diffusion difficult. A more well-behaved CF (see Fig. 2c), preserving this transition range whatever the $\kappa$ value, is the following:

$$
c_{3}(x, t)=\frac{1}{2}[\tanh (\gamma(\kappa-\|\nabla I(x, t)\|))+1],
$$

where $\gamma$ controls the steepness of the min-max transition region, whereas $\kappa$ controls the extent of the diffusion region in terms of gradient gray-level. In all our experiments, fixing $\gamma=0.2$ is proven to be efficient [16]. To substantiate all these observations, we use 


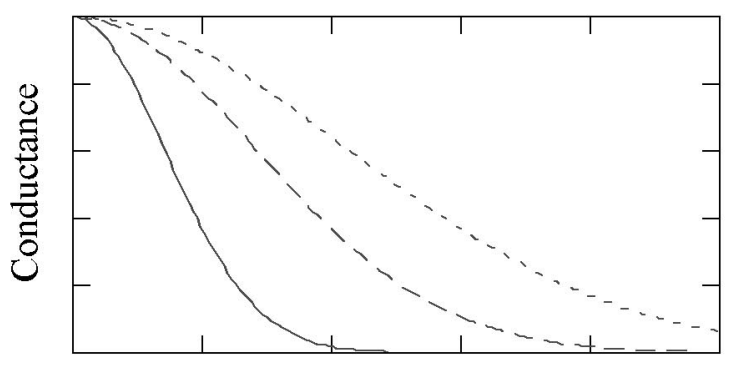

Gradient

(a)

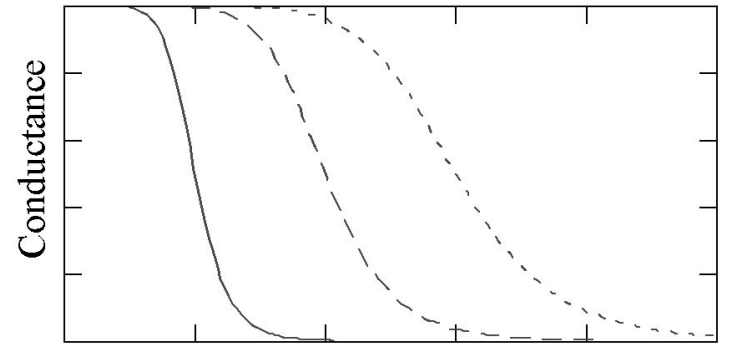

Gradient

(b)

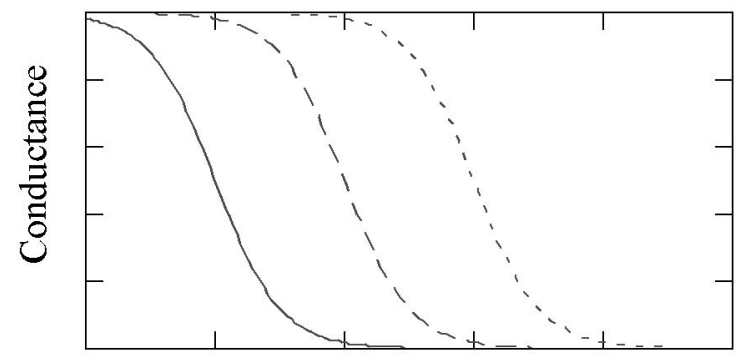

Gradient

(c)

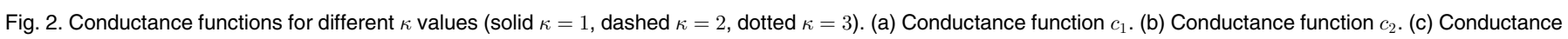
function $c_{3}$.

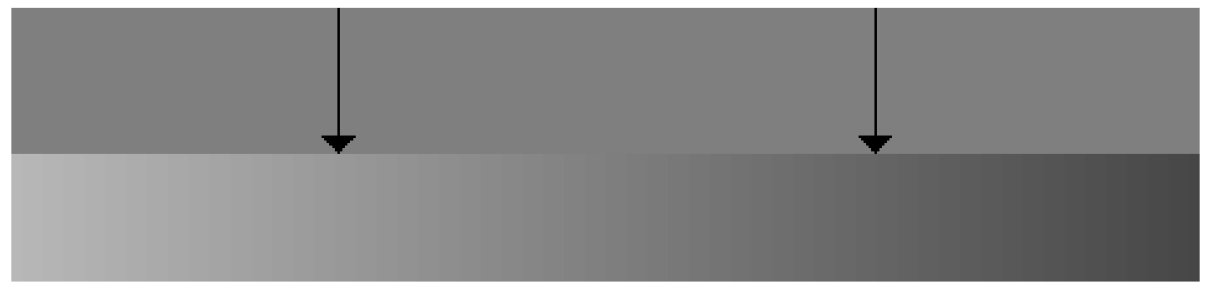

(a)

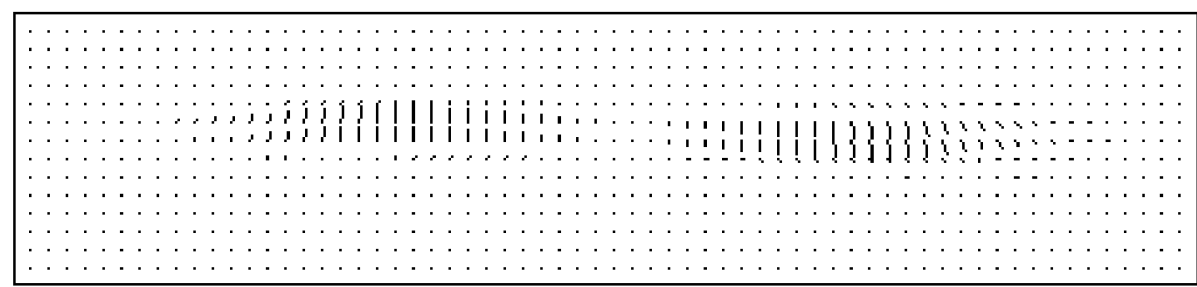

(b)

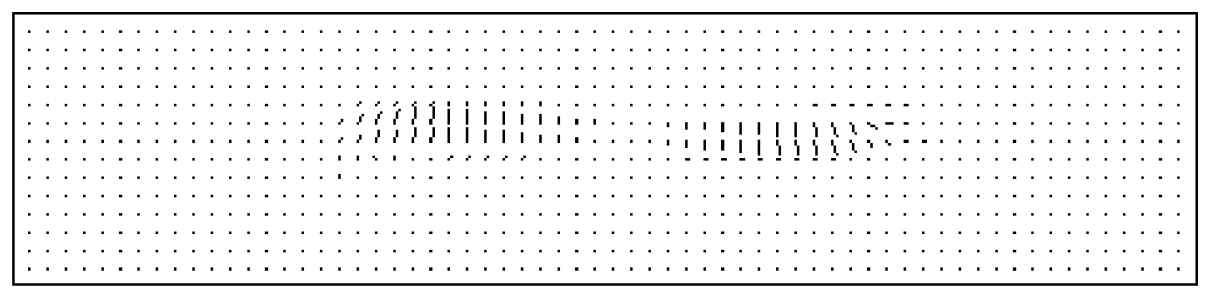

(c)

Fig. 3. The effect of the conductance function choice. (a) Test image (the arrows indicate the transition where the gradient gray-level is 20 (i.e., threshold $\kappa_{s}$ )). (b) Diffused field with $c_{1}$. (c) Diffused field with $c_{3}$. 


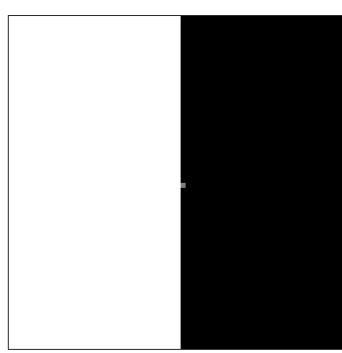

(a)

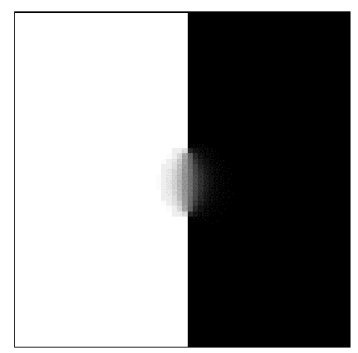

(c)

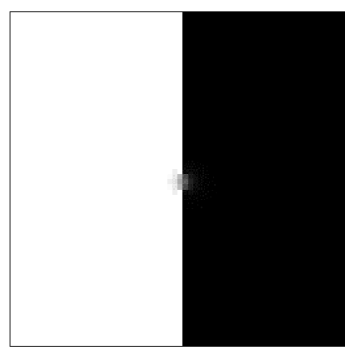

(b)

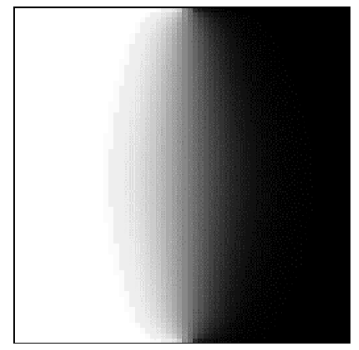

(d)
Fig. 4. Pinhole effect illustration. (a) Original image. (b) Result of SNAD after 25 iterations. (b) Result of SNAD after 50 iterations. (d) Result of SNAD after 150 iterations.

the synthesized image shown in Fig. 3a. The OF-GIC allows to track the effect of the diffusion when using the two CFs $c_{1}$ and $c_{3}$. In this experiment, $\mathrm{AD}$ is performed for 10 iterations with $\kappa=20$ and $\gamma=0.2$. Notice that the use of $c_{1}$ yields an ill-defined diffusion in high gradient regions leading to slow transition where initially the gradient reaches its maximum value (see Fig. 3b). Thus, diffusion continues even in sharp regions when using $c_{1}$, whereas, our function $c_{3}$ results in a well-defined sharp transition in these regions (see Fig. 3c).

\subsection{Pinhole Effect (PE)}

A new phenomenon PE is addressed in the following: It happens when a pixel or a group of adjacent pixels with a gray-level of intermediate value is near a sharp transition region. To illustrate $\mathrm{PE}$ in physics context, consider a medium where two or more regions with different conductivity are separated by interfaces. Then, it is assumed that a fluid can only diffuse inside regions, but never across frontiers. Now, if there exists a small hole on an interface, then the fluid can diffuse across these frontiers. In the image context, the pixel responsible of the PE will be called "vanishing point" (VP). When diffusion takes place, this point serves as a junction between the two regions leading to an undesirable smoothing effect through edges. To point out this effect, a synthetic image with a VP in the vicinity of a sharp edge is generated. Figs. $4 \mathrm{~b}, 4 \mathrm{c}$, and $4 \mathrm{~d}$ show the SNAD evolution after 25, 50, and 150 iterations, respectively. In order to avoid PE, a simple method is proposed and evaluated in the following: To localize VP we examine the gradient of the current pixel and those of its neighbors. Let $\mu_{-}, \mu_{+}$, and $\mu_{0}$ represent, respectively, the mean gray-level of pixels with negative flow, that of pixels with positive flow, and that of the central pixel. For a given gradient threshold value $\kappa_{s}$, the central pixel is considered as a VP if the following conditions are simultaneously satisfied:

$$
\left.\begin{array}{l}
0 \leq \mu_{0}-\mu_{-} \leq \kappa_{s} \\
0 \leq \mu_{+}-\mu_{0} \leq \kappa_{s} \\
\kappa_{s} \leq \mu_{+}-\mu_{-}
\end{array}\right\}
$$

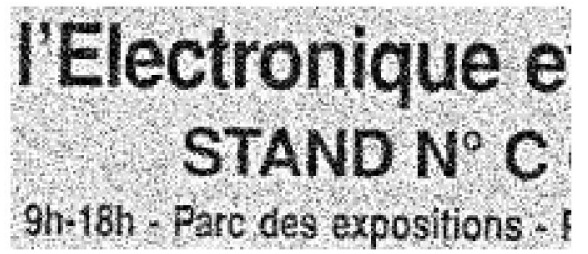

(a)

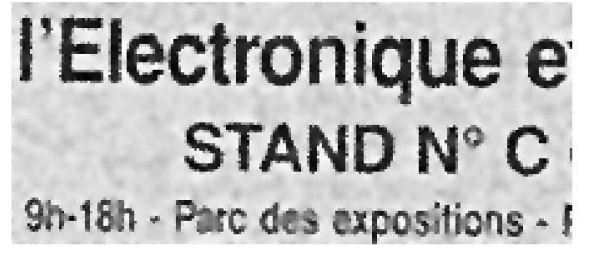

(c)

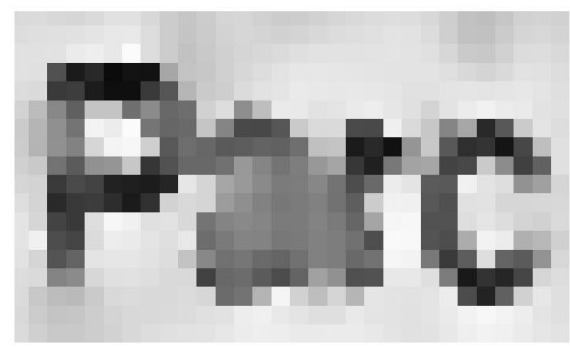

(e)

\section{l'Electronique e STAND $N^{\circ} \mathrm{C}$ 9h-18h - Parc des expositions - I}

(b)

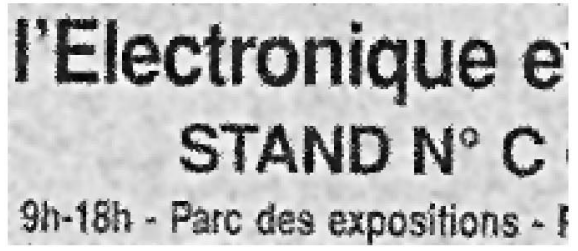

(d)

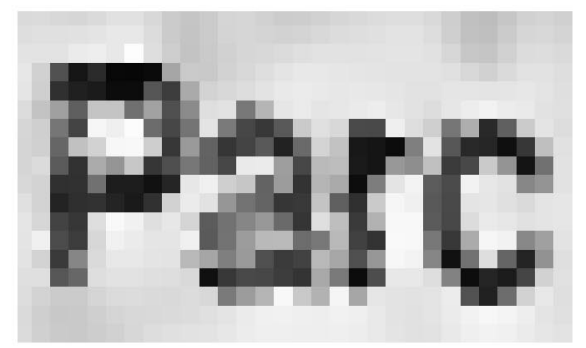

(f)

Fig. 5. Pinhole effect illustration in actual image: comparison of SNAD with ANAD. (a) Original noisy image. (b) SNAD with $c_{3}, \kappa_{2}=50$, after six iterations. (c) SNAD with $c_{3}, \kappa_{s}=100$, after six iterations. (d) ANAD with $c_{3}, \kappa_{s}=100$, after six iterations. (e) Zoomed region of interest of image (c). (f) Zoomed region of interest of image (d). 


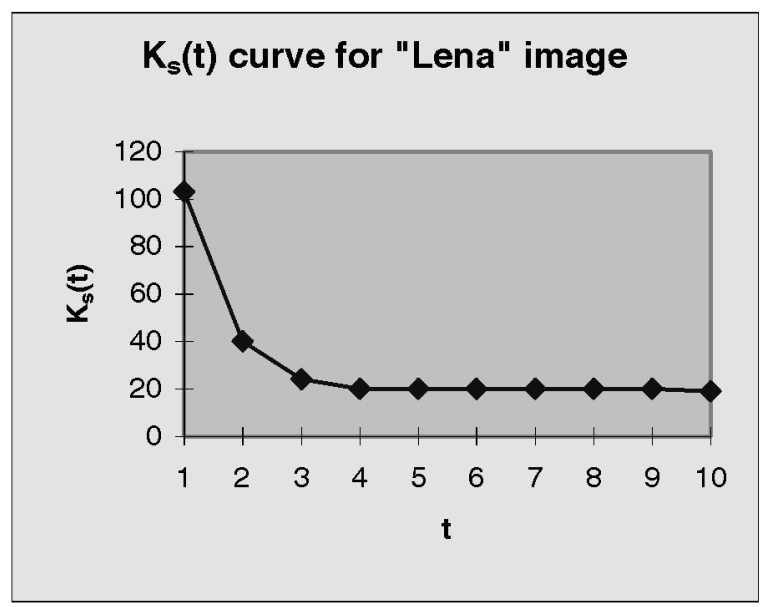

Fig. 6. Experimental $\kappa_{s}(t)$ curve.

The method of computing $\kappa_{s}$ will be addressed in the next section and an analytical proof of the existence of PE and the plausibility of conditions (13) is given in the Appendix. When VP is detected, the diffusion is stopped or the point is replaced by a neighbor. To analyze the PE in a realistic situation, we consider an actual image representing a text corrupted by an additive white gaussian noise (Fig. 5a) in order to create some VPs leading to PE. Fig. $5 \mathrm{~b}$ shows the result when applying SNAD with $c_{3}, \kappa_{s}=50$, and after six iterations. For this $\kappa_{s}$ value, the PE is not noticeable, but the noise is not completely removed. Increasing the $\kappa_{s}$ value will result in an image where the noise is totally smoothed out, but the PE is clearly visible, especially on the character contours, as shown in Fig. 5c, whereas Fig. 5d displays the result obtained when the PE is avoided using our criterion expressed in conditions (13). For subjective comparison, a region of interest in images Fig. $5 \mathrm{c}$ and Fig. $5 \mathrm{~d}$ is zoomed and shown in Figs. 5e and 5f, respectively.

\subsection{Edgeness Threshold Estimation}

The critical value $\kappa_{s}$ defines two different behaviors of the diffusion: blurring effect if $\|\nabla I\| \leq \kappa$ and edge sharpening for $\|\nabla I\|>\kappa_{s}$. The $\gamma$ parameter determines the length of the min-max diffusion range, i.e., the gradient range allowing varying the process from minimum (zero) diffusion to maximum diffusion. The proposed $\mathrm{CF} c_{3}$ allows adjusting the diffusion when the gradient threshold $\kappa_{s}$ is known. For this purpose, a simple effective method for estimating the gradient threshold is described in the following. In contrast with the approach of $\mathrm{Li}$ and Chen, where this parameter is empirically chosen to decrease as the diffusion evolves, we derive here a method making $\kappa_{s}$ signal dependent. To achieve that, we make use of two less restrictive assumptions:

- At least a fraction, say $\alpha$, of the image is composed of homogeneous regions (typically 10 percent).

- The random noise is identically and independently distributed in the image.

The image is subdivided into nonoverlapping blocks of the same size, typically between $4 \times 4$ and $32 \times 32$ pixels. A local homogeneity or uniformity measure [17] is then defined and

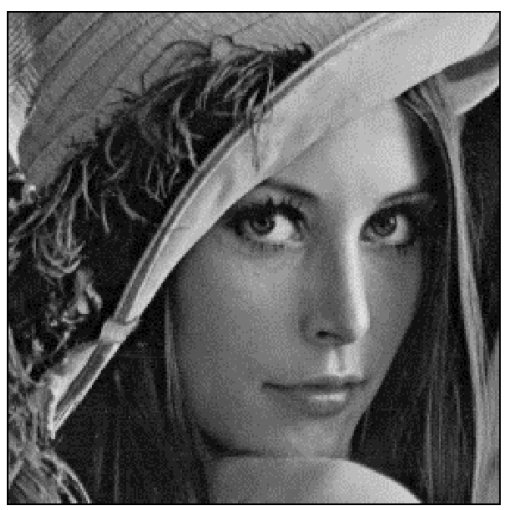

(a)

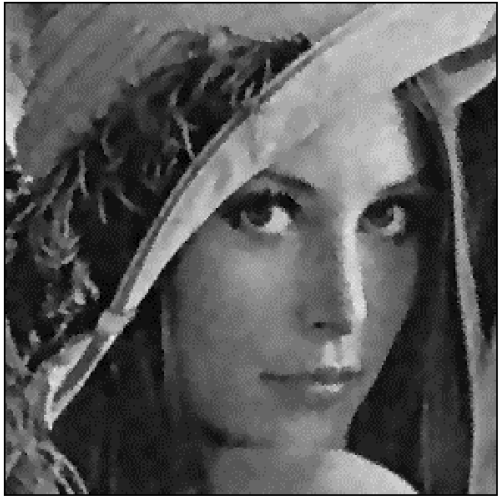

(d)

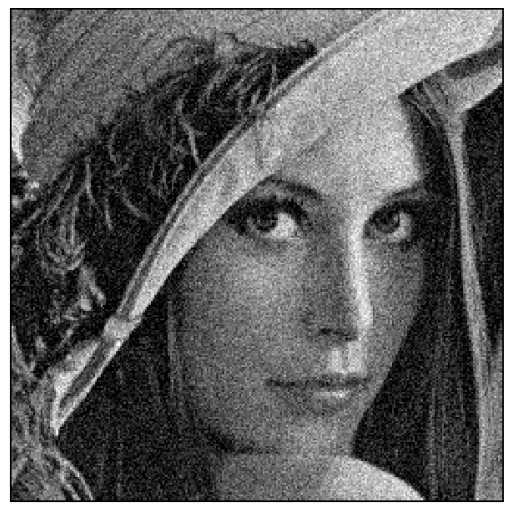

(b)

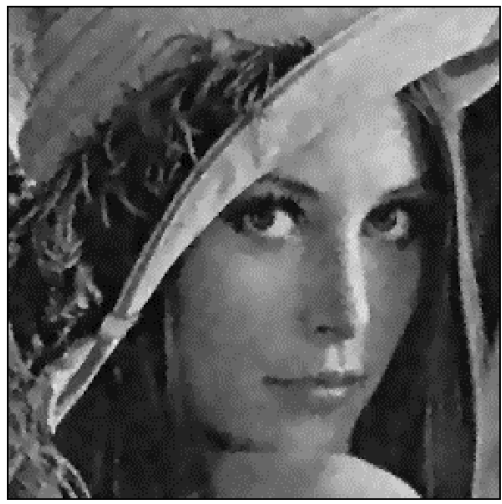

(e)

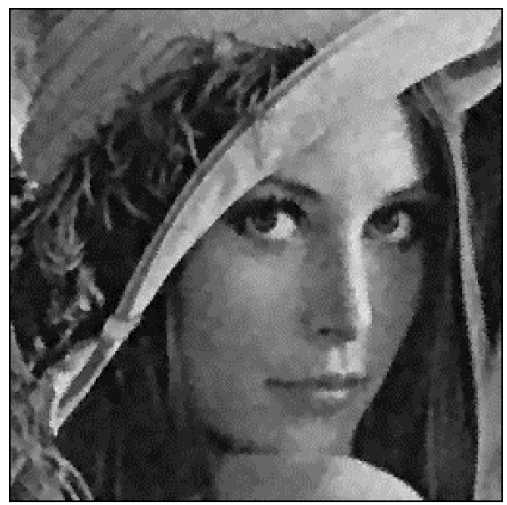

(c)

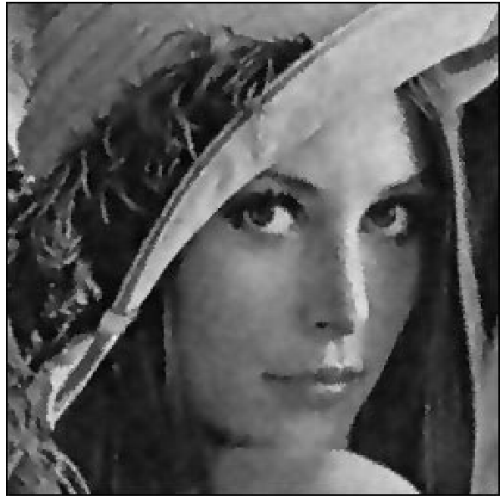

(f)

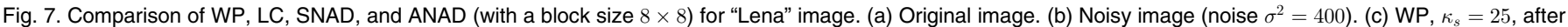

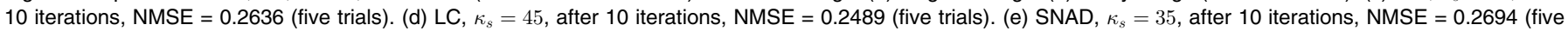
trials). (f) ANAD, after 10 iterations, NMSE $=0.2323$ (one trial). 
measured in each block. Then, the blocks are sorted in order of decreasing homogeneity. Only a fraction of the blocks with high homogeneity measure are retained. They represent then the homogeneous regions of the image, as assumed in our first hypothesis. Now, we use the second hypothesis as an argument to estimate the noise variance. Indeed, since the noise is assumed to be randomly distributed in the image space, then a practical way to estimate its variance is to consider homogeneous regions where small variations or texture are mainly due to noise. Thus, in order to estimate $\kappa_{s}$, we use, as Perona and Malik, a noise estimator similar to that described in the edge detection method of Canny [18]. But, in contrast with these authors, only selected homogeneous blocks are taken into account in this estimation. This analysis is performed at each iteration and a new threshold $\kappa_{s}$ value is automatically determined. It could be noticed in Fig. 6 that the curve representing the evolution of this parameter shares the same decreasing behavior than that of $\mathrm{Li}$ and Chen. But, these authors do not explain how to adapt $\kappa_{s}$ to the signal local characteristics during the process. Here, a solution for automatically adapting $\kappa_{s}$ at each iteration is proposed such as to make $\kappa_{s}$ signal dependent.

\section{EXPERimental EVALUATION OF ANAD}

To evaluate the efficiency of ANAD method, actual images corrupted by Gaussian white noise are considered. Our method is compared with the other cited methods. For subjective comparison only, visual judgment is used, whereas, for objective comparison, we use the Normalize Mean Square Error (NMSE) given by:

$$
N M S E=\sum_{x, y}\left[I_{r}(x, y)-I_{0}(x, y)\right]^{2} / \sum_{x, y}\left[I_{d}(x, y)-I_{0}(x, y)\right]^{2},
$$

where $I_{0}, I_{d}$, and $I_{r}$ are the original, the degraded, and the filtered signal, respectively. The standard Lena image (Fig. 7a) is used for comparison. This image is corrupted by Gaussian noise and treated by the four methods referenced WP for Whitaker-Pizer, LC for Li-Chen, SNAD for Perona-Malik, and ANAD for our method. The first three methods are applied with the optimum, on the basis of NMSE and visual quality criteria, value of the conductance parameter $\kappa_{s}$ obtained after five trials. Through Figs. $7 \mathrm{c}, 7 \mathrm{~d}, 7 \mathrm{e}$, one can observe that the results of applying these four methods are, on the basis of visual quality criterion, slightly comparable, whereas, on the basis of NMSE criterion, ANAD outperforms the other compared methods.

\section{Conclusion}

Through this study it is demonstrated that the method presented here is an improvement of the nonlinear anisotropic diffusion filtering. It has been shown that it is possible to obtain an adaptive and automatic AD with some simple assumptions about the signal and the noise. It is proven that the use of OFT with GIC as a tool for tracking the process may help in understanding the diffusion in image signal. Furthermore, a new CF sharing some interesting properties has been introduced. It is also proven that, even when the $\mathrm{CF}$ is well understood, this nonlinear filtering can produce unexpected results, such as PE. In summary, the superiority of the proposed method over the others is essentially its adaptability and flexibility. Indeed, in our method, the only parameter to chose is the size of the block analysis. It is easier to have an intuitive idea about the size of the homogeneous block than to select the adequate value for $\kappa_{s}$ after many trials, as done in the other cited methods. Some opened questions are being examined in the near future. It essentially concerns the strategy to adopt for providing a low computational cost and adapting the block size during the iterative process evolution.

\section{APPENDIX}

Here, for the sake of simplicity, the PE in the case of 1D discrete signal is analyzed. Let $I_{i}$ be the intensity of the point $i, I_{i-1}$, and $I_{i+1}$ those of its left and right neighbors, respectively. First, let us assume this transition such that $\left\|I_{i+1}-I_{i}\right\|>\kappa_{s}$. Then, the diffusion could not take place in this case. Now, let us consider a simple situation where the intensity of the center point at $i$ is such that $I_{i}=\left(I_{i+1}+I_{i-1}\right) / 2$. Thus, this point is a VP since the conditions expressed in (13) are fulfilled. Indeed, $0 \leq I_{i}^{0}-I_{i+1}^{0} \leq \kappa_{s}, 0 \leq I_{i-1}^{0} \leq \kappa_{s}$, and $\kappa_{s} \leq I_{i-1}^{0}-I_{i+1}^{0}$, where the superscript 0 stands for the initial state. If we call $c_{E i}$ and $c_{W i}$ the conductance coefficients (East and West, resp.), the iterative version of (1) can be written in the following form:

$$
I_{i}^{t+1}=I_{i}^{t}+\lambda\left[c_{E} \delta_{E} I+c_{W} \delta_{W} I\right]_{i}^{t},
$$

where $t$ is the temporal parameter, $\lambda \in[0,1 / 3]$ to insure the stability of the iterative scheme and the nearest-neighbor difference operators are: $\delta_{E} I_{i} \equiv I_{i+1}-I_{i} \quad$ and $\quad \delta_{W} I_{i} \equiv I_{i-1}-I_{i}$. The conductance coefficients are updated every iteration as done in [8]: $c_{E_{i}}^{t}=c\left(\left\|\nabla I_{i+1 / 2}^{t}\right\|\right)$ and $c_{W_{i}}^{t}=c\left(\left\|\nabla I_{i-1 / 2}^{t}\right\|\right)$.

Given the form of the considered signal the left and right discrete differences are the same: $\left\|\nabla I_{i+1 / 2}^{0}\right\|=\left\|\nabla I_{i-1 / 2}^{0}\right\|$, i.e., $c_{E_{i}}^{0}=$ $c_{W_{i}}^{0} \equiv c_{i}^{0}$ and $I_{i-1}^{0}-I_{i}^{0}=I_{i}^{0}-I_{i+1}^{0} \equiv \Delta_{i}^{0}$, where $\Delta_{i}^{0}>0$.

The use of (A.1) at the first iteration yields:

$$
\begin{aligned}
I_{i}^{1} & =I_{i}^{0}+\lambda\left[c_{E} \delta_{E} I+c_{W} \delta_{W} I\right]_{i}^{0} c \\
& =I_{i}^{0}+\lambda\left[c_{i}^{0}\left(I_{i+1}^{0}-I_{i}^{0}\right)+c_{i}^{0}\left(I_{i-1}^{0}-I_{o}^{0}\right)\right]_{i}^{0} \\
& =I_{i}^{0}+\lambda c_{i}^{0}\left[-\Delta_{i}^{0}+\Delta_{i}^{0}\right]=I_{i}^{0} \\
I_{i-1}^{1} & =I_{i-1}^{0}+\lambda c_{E_{i-1}}^{0} \delta_{E} I_{i-1}^{0} \\
& =I_{i-1}^{0}+\lambda c\left(\left\|\nabla I_{i-1 / 2}^{0}\right\|\right)\left(I_{i}^{0}-I_{i-1}^{0}\right) \\
& =I_{i-1}^{0}-\lambda c_{i}^{0} \Delta_{i}^{0} \\
I_{i+1}^{1} & =I_{i+1}^{0}+\lambda c_{W_{i+1}}^{0} \delta_{W} I_{i+1}^{0} \\
& =I_{i+1}^{0}+\lambda c\left(\left\|\nabla I_{i+1 / 2}^{0}\right\|\right)\left(I_{i}^{0}-I_{i+1}^{0}\right) \\
& =I_{i+1}^{0}+\lambda c_{i}^{0} \Delta_{i}^{0} .
\end{aligned}
$$

This result shows that the VP $i$ is not affected by the diffusion and that the same amount of quanta, in the framework of the CIM, is received or emitted by the right and left neighboring cells, respectively. In terms of intensity, it corresponds to a gray-level variation of $\lambda c_{i}^{0} \Delta_{i}^{0}$ (i.e., PE).

\section{ACKNOWLEDGMENTS}

The authors wish to thank the anonymous reviewers for their helpful comments and criticisms in improving the earlier versions of this paper.

\section{REFERENCES}

[1] A.C. Bovik, T.S. Huang, and D.C. Munson Jr., "The Effect of Median Filtering on Edge Estimation and Detection," IEEE Trans. Pattern Analysis and Machine Intelligence, vol. 9, pp. 181-194, 1987.

[2] L. Yin, R. Yang, M. Gabbouj, and Y. Neuvo, "Weighted Median Filters: A Tutorial," IEEE Trans. Circuits, Systems Processing, vol. 43, no. 3, pp. 157-192, 1996.

[3] J. Serra, Image Analysis and Mathematical Morphology. New York: Academic Press, 1982. 
[4] P. Maragos and R.W. Schafer, “Morphological Filters-Part I: Their SetTheoretic Analysis and Relations to Linear Shift-Invariant Filters," IEEE Trans. Acoustics, Speech, and Signal Processing, vol. 35, no. 8, pp. 1,153-1,169, 1987.

[5] P. Perona and J. Malik, "Scale Space and Edge Detection Using Anisotropic Diffusion," Proc. IEEE Workshop Computer Vision, pp. 16-22, Miami, Nov. 1987.

[6] L. Alvarez, P.L. Lions, and J.M. Morel, "Image Selective Smoothing and Edge Detection by Nonlinear Diffusion (II)," SIAM J. Numerical Analysis, vol. 29, pp. 845-866, 1992.

[7] V. Caselles, J.M. Morel, G. Sapiro, and A. Tannenbaum, “Introduction to the Special Issue on Partial Differential Equations and Geometry-Driven Diffusion in Image Processing," IEEE Trans. Image Processing, vol. 7, no. 3, pp. 269-273, 1998.

[8] P. Perona and J. Malik, "Scale-Space and Edge Detection Using Anisotropic Diffusion," IEEE Trans. Pattern Analysis and Machine Intelligence, vol. 12, no. 7, pp. 629-639, July 1990.

[9] F. Catté, P.L. Lions, J.M. Morel, and T. Coll, "Image Selective Smoothing and Edge Detection by Nonlinear Diffusion," SIAM J. Numerical Analysis, vol. 29, no. 1, pp. 182-193, 1992.

[10] R.T. Whitaker and S.M. Pizer, "A Multi-Scale Approach to Non Uniform Diffusion," CVGIP: Image Understanding, vol. 57, no. 1, pp. 99-110, 1993.

[11] X. Li and T. Chen, "Nonlinear Diffusion with Multiple Edginess Thresholds," Pattern Recognition, vol. 27, no. 8, pp. 1,029-1,037, 1994.

[12] K.P. Horn and B.G. Schunck, "Determining Optical Flow," Artificial Intelligence, no. 17, pp. 185-203, 1981.

[13] J.L. Barron, D.J. Fleet, and S.S. Beauchemin, "Systems and Experiment Performance of Optical Flow Techniques," Int'l J. Computer Vision, vol. 12, no. 1, pp. 43-77, 1994.

[14] B.G. Schunck, "The Motion Constraint Equation for Optical Flow," Proc. Int'l Conf. Pattern Recognition, pp. 20-22, Montréal, Quebec, Canada, 1984.

[15] E.L. Cussler, Diffusion Mass Transfer in Fluid Systems. Cambridge Univ. Press, 1985.

[16] J. Monteil, "Traitement et Analyse par une Technique Adaptée de Flux Optique du Champ de Déformation de Matériaux à Partir de Séquences d'Images de Microscopie," PhD thesis, Université Paris VII, Mar. 1998.

[17] M.D. Levine and A.M. Nazif, "Dynamic Measurement of Computer Generated Image Segmentation," IEEE Trans. Pattern Analysis and Machine Intelligence, vol. 7, pp. 155-164, 1985.

[18] J. Canny, "A Computational Approach to Edge Detection," IEEE Trans. Pattern Analysis and Machine Intelligence, vol. 8, pp. 679-698, 1986. 